\title{
Excess Molar Volumes, Apparent Molar Volumes, Partial Molar Volumes of Methionine, An Amino Acid, in Water + Ethanol And Water + Methanol Solutions at $298.15 \mathrm{~K}$
}

\author{
Ufuk Sancar Vural*1, Saban Uysal ${ }^{2}$, \\ *1Pasabayır Mh. Mehmetcik Cd. 77/16, Bandirma, Balikesir, Turkey \\ ${ }^{2}$ Karabuk University, Faculty of Science, Department of Chemistry, Karabuk, Turkey \\ E-mail: ${ }^{1}$ usvural@gmail.com, ${ }^{2}$ sabanuysal@karabuk.edu.tr
}

Received 26 October 2021, Revised 21 December 2021, Accepted 10 January 2022

\begin{abstract}
Methionine is an amino acid that is extremely important for human health. To better understand the biochemical events occurring in the human body, the excess molar properties of methionine and aqueous ethanol and aqueous methanol mixtures were determined at $298.15 \mathrm{~K}$. Interactions between components in solutions are explained. Negative deviations from the ideal state have been observed in methionine solutions due to hydrogen bonds, dipole interactions, charge-transfer interactions.
\end{abstract}

Keywords: Methionine; excess molar volume; excess partial molar volume; apparent molar volume

\section{Introduction}

Methionine is an amino acid found in many proteins present in our bodies and food (Figure 1). It is not just a building block for proteins but contains some unique properties. The most important of these properties is its ability to be converted into sulfur-containing molecules [1]. Sulfur-containing molecules play an important role in protecting tissues, modifying DNA, and maintaining the proper functioning of cells $[2,3,4[$. Of the amino acids used to make protein in the body, only methionine and cysteine contain sulfur. Methionine also plays a critical role in initiating the process of making new proteins in cells. It occurs continuously as old proteins break down [5]. For example, it helps muscles produce new proteins after a muscle-damaging workout [6,7].<smiles>CSCCC(N)C(=O)O</smiles>

Figure 1. Methionine structure

Zhang et al. (2016) observed that the solubility of methionine in water is higher than in organic solvents, and the solubility decreases in water + acetone $<$ water + ethanol $<$ water + methanol in water + organic solvent mixtures [8]. According to the like dissolves like principle, they stated that the solubility of the amino acid, which has a polar structure, increases due to the increase in the polarity of organic solvents. Tyunina et al. (2020) reported that electrostatic interactions and the formation of hydrogen bonds were effective on the volumetric properties of methionine aqueous solutions [9]. El-Dossoki (2018) observed that the molal solubility of methionine decreases as the mole fraction of methanol and ethanol increases due to the salt effect [10]. He also determined that the apparent molar volume of DLmethionine in water and methanol-water and ethanol-water solutions increased as the mole fraction of methanol and ethanol increased. He pointed out the inverse proportion between volume and density as the reason for this.

The excess molecular properties of amino acid solutions are very useful for understanding the conformational stability and unfolding behaviour of protein molecules. Some researchers have used thermodynamic methods to investigate the interactions of amino acids with organic molecules or salts in aqueous solutions $[11,12]$.

Excess properties can be defined as the difference between the ideal volumetric properties of the mixture and the observed volumetric properties as a result of the molecular interactions between the components that make up the mixture and in the solution [13]. The excess volumes $\left(\mathrm{V}^{\mathrm{E}}\right)$ are defined as the difference of volume of real solutions and volume of pure components (ideal solution).

Excess thermodynamic properties, which can also be understood as deviations from ideal thermodynamic behaviours, provide a better understanding of the behaviour of components in chemical and biochemical events, and their roles in production and application processes. In general, the excess properties result from three types of interactions between the constituent molecules of liquid mixtures [14$15]$.

(a) Positive effects: Physical interactions consisting of dispersion forces or weak dipole-dipole interaction,

(b) negative effects: chemical or specific interactions, including charge transfer, H-bonds, and other complex formation interactions, 
(c) Structural effects: These are the structural contributions arising from the differences in the size and shape of the component molecules in the mixture depending on the structure of their molecules.

In this study, excess molar volumes, apparent molar volumes, partial molar volumes and excess partial molar volumes of the binary mixture of methionine + methanol and methionine + ethanol in water at $298.15 \mathrm{~K}$, which is one of the most important building blocks of proteins, were investigated.

\section{Materials}

Amino acids; methionine of $99.5 \%$ purity, methanol and ethanol of $99 \%$ purity were obtained from Merck Company. Some physicochemical properties of the components are given in Table 1.

Using twice-distilled water with a conductivity of 0.038 $\mu \mathrm{S} / \mathrm{cm}, 10 \mathrm{~cm}^{3}$ of alcohol solutions were prepared between $0-1$ mole fractions by increasing the volume by $0.5 \mathrm{~cm}^{3}$. To alcohol solutions, amino acid was added until saturation. The sample tubes were mixed with a magnetic stirrer overnight in a thermostatic water bath at $298.15 \pm 0.1 \mathrm{~K}$ by closing the mouth with a Teflon cap. Then, the solution was left to rest overnight and the supernatant was separated by filtration. The filtered solutions were kept in a thermostatic water bath at $298.15 \pm 0.1 \mathrm{~K}$ and their densities were measured by Anton Paar DMA 4500 Denismeter. Experiments were performed in 4 repetitions.

Table 1 Molecular weight and densities of pure components in mixtures

\begin{tabular}{lcc}
\hline Component & M, g/mol & Density, 298.15 K \\
\hline Methionine & 149.21 & Powder \\
Methanol & 32.04 & 0.7048 \\
Ethanol & 46.07 & 0.7875 \\
Water & 18.02 & 0.9968 \\
\hline
\end{tabular}

\section{Methodology}

\subsection{Excess Molar Properties}

The excess molar volume of a liquid solution is defined by the following equation [16-17].

$V^{E}=V-\sum_{i=0} x_{i} V_{i}^{0}$

where $V$ is the molar volume of the solution, $x i$ is the mole fractions of components, and $V_{i}{ }^{0}$ is the molar volumes of pure components, respectively. Eq. (1) can be written in terms of density as Eq. (2). The excess molar volumes, calculated from the density data in Eq. (4), are listed in Table 3 and plotted in Figure 3.

$V^{E}=\sum_{i=1} X_{i} M_{i}\left(\frac{1}{\rho}-\frac{1}{\rho_{i}}\right)$

in which $M_{i}$ is the molar masses of components, $\rho$ is the density of the mixture, and $\rho_{i}$ represent the densities of pure components, respectively.

The excess molar properties $\left(\mathrm{V}^{\mathrm{E}}\right)$ can be correlated using the Redlich-Kister equation [16-20]:

$V^{E}=X_{i}\left(1-X_{i}\right) \sum_{i=1} A_{i}\left(2 X_{i}-1\right)^{i}$

The values of the coefficients $A_{i}$ were calculated by the method of least squares along with the standard deviation
$\sigma\left(V^{E}\right)$. The coefficients $A_{i}$ are adjustable parameters for a better fit of the excess functions.

$\sigma\left(V^{E}\right)=\left[\frac{\sum_{i=1}^{i=n}\left(V_{c a l, i}^{E}-V_{\text {exp }, i}^{E}\right)}{n}\right]^{1 / 2}$

where $n$ is the number of parameters, $V_{\text {expt }}$ and $V_{\text {cal }}$ are the experimental and calculated parameters, respectively.

\subsection{Partial Volume and Excess Partial Volume}

To have more knowledge about interactions solute and solvent, the values of partial molar volumes of binary mixtures have been calculated using the following equations [18-20]:

$V_{i}=\left(\frac{\partial V}{\partial n_{i}}\right)_{P, T, n_{j}}$

Differentiation of Eq. (3) and combination with Eq. (5), gives the following equations for the partial molar volume of pure components

$V_{1}=V^{E}+V_{1}^{0}+\left(1-X_{1}\right)\left(\partial V^{E} / \partial X_{1}\right)_{T, P}$

$V_{2}=V^{E}+V_{2}^{0}-X_{1}\left(\partial V^{E} / \partial X_{1}\right)_{T, P}$

where $V_{1}^{0}$ and $V_{2}^{0}$ are the molar volume of the pure component. Combination of Eqs. (3), (6) and (7) leads to Eqs. (8) and (9).

$V_{1}=V_{1}^{0}+(1-x)^{2} \sum_{i=0} A_{i}(1-2 x)^{i}-2 x(1-$

$x)^{2} \sum_{i=1} A_{i} i(1-2 x)^{i-1}$

$V_{2}=V_{2}^{0}+x^{2} \sum_{i=0} A_{i}(1-2 x)^{i}+2 x^{2}(1-$

x) $\sum_{i=1} A_{i} i(1-2 x)^{i-1}$

Values of the partial molar volumes at infinite dilution, $V_{i}^{\infty}$, were obtained by the linear extrapolation of corresponding partial molar volumes using Eq. (8) and Eq. (9). Extrapolation of $V_{i}$ to $x_{i}=0$ results in $V_{i}^{\infty}$. The excess partial molar volumes at infinite dilution, $V_{i}^{E}$, were calculated using the following relations [18-20]:

$V_{i}^{E}=V_{i}-V_{i}^{0}$

The partial properties at infinite dilution are of interest since, at the limit of infinite dilution, the (solute + solute) interactions disappear. The values of the partial molar volume at infinite dilution provide information about (solute + solvent) interaction, independent of the composition effect. Setting $(x=0)$ in Eq. (8) and $(x=1)$ in Eq. (9) leads to Eq. (11) and Eq. (12). Eq. (11) and (12) give $V_{1}^{\infty}$ and $V_{2}{ }^{\infty}$ (the partial molar volumes at infinite dilution) for the component (1) and (2), respectively [18-20].

$V_{1}^{\infty}=V_{1}^{0}+\sum_{i=0} A_{i}(-1)^{i}$

$V_{2}^{\infty}=V_{2}^{0}+\sum_{i=0} A_{i}$

Rearrangement of Eqs. (11) and (12) leads to Eqs. (13) and (14) for the excess partial molar volumes of components at infinite dilution, 


$$
\begin{aligned}
& V_{1}^{E, \infty}=\sum_{i=0} A_{i}(-1)^{i} \\
& V_{2}^{E, \infty}=\sum_{i=0} A_{i}
\end{aligned}
$$

Eq. (13) and Eq. (14) represent the partial molar volumes of components in solutions at infinite dilution, respectively.

\subsection{Apparent Molar Volume}

The apparent molar volume $\left(V_{\varphi}\right)$ is a very useful parameter to understand the interactions between ionsolvent, ion-ion and solvent-solvent (structural) molecules that occur in mixtures. The Redlich-Kister equation and its derivatives do not always provide the best representation of properties of either component at infinite dilution in the other component. Instead of using the Redlich-Kister equation, we have also considered another approach, which may be more convenient and accurate, by calculating the partial molar volume at infinite dilution through apparent molar volumes. Apparent molar volumes of components, $V \phi$, were calculated from experimental data using the following relations $[18-20]$.

$V_{\emptyset 1}=\frac{\left(V-x_{2} V_{2}^{0}\right)}{x_{1}}=\frac{M_{1}}{\rho}+\frac{\left(\rho_{2}-\rho\right) x_{2} M_{2}}{x_{1} \rho \rho_{2}}$

$V_{\emptyset 2}=\frac{\left(V-x_{1} V_{1}^{0}\right)}{1-x_{1}}=\frac{M_{2}}{\rho}+\frac{\left(\rho_{1}-\rho\right) x_{1} M_{1}}{x_{2} \rho \rho_{1}}$

where $M_{i}, m, \rho_{i}$ and $\rho_{i}^{0}$ are molecular weight, molality, densities of components and pure components, respectively. Extrapolation of $V_{\emptyset i}$ to $x_{i}=0$ give the values of the limiting apparent molar volume $\left(V_{\emptyset, i}^{0}\right)$. The excess apparent molar volumes at infinite dilution, $V_{\emptyset, i}^{E}$, were also calculated by equations similar to Eqs. (10). The apparent molar volumes of $V_{\phi 1}$ and $V_{\phi 2}$ can be calculated from Eq. (17) and Eq. (18).

$V_{\emptyset 1}=\frac{V-(1-x) V_{2}^{0}}{x}$

$V_{\emptyset 2}=\frac{V-x V_{1}^{0}}{1-x}$

where $V_{1}^{0}$ and $V_{2}^{0}$ are the molar volumes determined from the experimental densities by $M_{i} / \rho_{\mathrm{i}}$, and $V$ is the molar volume of the solution determined by the following equation:

$\mathrm{V}=\mathrm{V}^{\mathrm{E}}+\left(\mathrm{x}_{1} V_{1}^{0}+\mathrm{x}_{2} V_{2}^{0}\right)$

The combination of Eq. (1), Eq. (17), and Eq. (18) lead to:

$V_{\emptyset 1}=V_{1}^{0}-\frac{V^{E}}{x}$

$V_{\emptyset 2}=V_{2}^{0}-\frac{V^{E}}{1-x}$

Simple graphical or analytical extrapolation of $V_{\phi 1}$ to $x=0$ and $V_{\phi 2}$ to $x=1$ leads to the partial molar volumes at infinite dilution, $V_{1}^{\infty}$ and $V_{2}^{\infty}$, respectively. Further, the limiting partial molar volume is expressed as $V_{i}^{\infty}=V_{i}^{E, \infty}+V_{i}^{0}$ from which the $V_{i}^{E, \infty}$ can be derived. It can be observed that the procedures of obtaining $V_{i}^{E, \infty}$ values by Eq. (13) and (14) or from the extrapolated $V_{\phi I}$ values of Eq. (20) and Eq. (21) lead to comparable magnitudes of $V_{i}^{\infty}$ values.
Partial molar volumes were calculated at infinite dilution from excess molar volumes using a method based on extrapolation of reduced volume. This method was obtained by rearrangement of Eq. (20) and division by (1-x).

$\frac{V^{E}}{x(1-x)}=\frac{V_{\emptyset i}-V_{i}^{0}}{1-x}$

Linear extrapolation of the "reduced volume" represented by $V^{E} / x(1-x)$ to $x=0$ and $x=1$ leads to the desired $V_{1}^{\infty}$ and $V_{2}^{\infty}$, respectively. Thus, the methods of obtaining $V_{1}^{\infty}$ and $V_{2}^{\infty}$ by way of Eqs. (14) and (15), extrapolation of $V_{\phi i}$ to $x=0$ or $V^{E} / x(1-x)$ to $x=0$ are all satisfactory, giving equally approximately equal values of partial molar volumes at infinite dilution.

\section{Result and Discussion}

In this study, the densities, apparent molar volumes, partial molar volumes and excess molar properties of saturated solutions of methionine in aqueous solutions of different concentrations of methanol and ethanol at a constant temperature of $298.15 \mathrm{~K}$ were investigated. The densities of aqueous mixtures of amino acid over the entire range of compositions at 298.15 are shown in Table 2 and Figure 2.

Table 2. Densities and Excess Molar Volumes of Methionine + Ethanol + Water) and (Methanol + Water) Solutions at $298.15 \mathrm{~K}$.

\begin{tabular}{cllll}
\hline & \multicolumn{3}{l}{ Densities, $\left(\mathrm{g} / \mathrm{cm}^{3}\right)$} & $\mathrm{V}^{\mathrm{E}},\left(\mathrm{cm}^{3} / \mathrm{mol}\right)$ \\
\cline { 2 - 5 }$x$ & Methanol & $\begin{array}{l}\text { Ethanol } \\
+ \text { Water }\end{array}$ & $\begin{array}{l}\text { Methanol } \\
+ \text { Water }\end{array}$ & $\begin{array}{l}\text { Ethanol } \\
+ \text { Water }\end{array}$ \\
\hline 0.00 & 0.9964 & 0.9983 & 0.0000 & 0.0000 \\
0.05 & 1.0079 & 0.9951 & -0.6118 & -0.6359 \\
0.10 & 1.0141 & 0.9901 & -1.1343 & -1.2238 \\
0.15 & 1.0143 & 0.9837 & -1.5474 & -1.7660 \\
0.20 & 1.0085 & 0.9765 & -1.8410 & -2.2658 \\
0.25 & 0.9973 & 0.9687 & -2.0135 & -2.7269 \\
0.30 & 0.9818 & 0.9606 & -2.0715 & -3.1528 \\
0.35 & 0.9635 & 0.9524 & -2.0306 & -3.5466 \\
0.40 & 0.9438 & 0.9442 & -1.9161 & -3.9098 \\
0.45 & 0.9245 & 0.9359 & -1.7630 & -4.2420 \\
0.50 & 0.9070 & 0.9276 & -1.6136 & -4.5397 \\
0.55 & 0.8925 & 0.9190 & -1.5135 & -4.7954 \\
0.60 & 0.8819 & 0.9100 & -1.5035 & -4.9969 \\
0.65 & 0.8755 & 0.9002 & -1.6096 & -5.1255 \\
0.70 & 0.8728 & 0.8892 & -1.8329 & -5.1549 \\
0.75 & 0.8726 & 0.8766 & -2.1397 & -5.0486 \\
0.80 & 0.8727 & 0.8617 & -2.4549 & -4.7577 \\
0.85 & 0.8696 & 0.8440 & -2.6535 & -4.2159 \\
0.90 & 0.8587 & 0.8227 & -2.5449 & -3.3335 \\
0.95 & 0.8340 & 0.7970 & -1.8311 & -1.9866 \\
1.00 & 0.7877 & 0.7661 & 0.0000 & 0.0000 \\
\hline
\end{tabular}

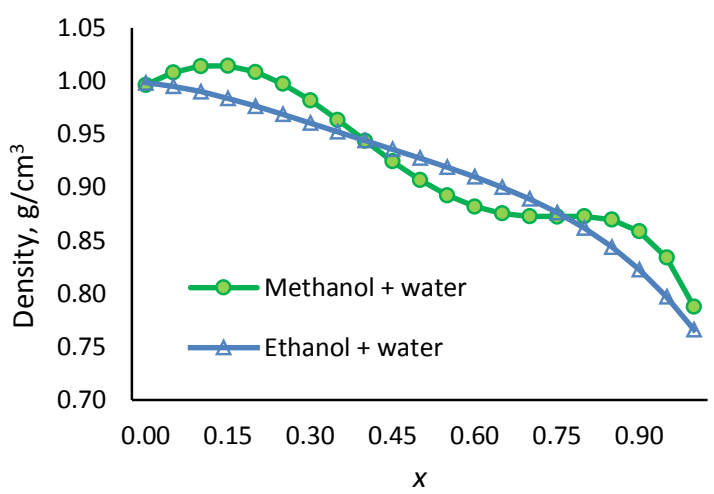

Figure 2. Densities of solutions of methionine + (ethanol +water) and (methanol + water) at $298.15 \mathrm{~K}$. 
As can be seen from Table 2, the solubility of amino acids decreases as the alcohol concentration decreases. Therefore, densities of binary mixtures of methionine and alcohol decrease as methanol and ethanol mole fraction increase. The density of the ethanol solution decreases slightly more than the methanol solution. However, the densities of the binary mixtures were found to be close to each other due to the strong interaction between functional groups in the side chain of the polar methionine and both alcohols.

The excess molar properties of methionine in aqueous alcohol solutions deviate from the ideal state. Chemical, physical, structural interactions between solvent-solute and components are the cause of this deviation. As can be seen in Table 2 and Figure 3, the excess molar volume of the binary mixture of methionine with ethanol and methanol showed a negative deviation. Negative deviation in excess molar property is in the direction of increasing alcohol fraction. The negative deviation of excess molar volume in ethanol solution is greater and more regular because ethanol is more polar than methanol and forms stronger $\mathrm{H}$-bonds. The excess molar volume in methanol solutions shows irregularly negative deviations. The tendency of methionine to dissolve in the more polar water is deactivated as the mole fraction of methanol increases. Negative deviations in the excess molar volume of a binary mixture of methionine and both alcohol solutions indicate a strong hydrogen bond and chemical effects between the solvent and solute. Thus, it was understood that chemical interactions including charge transfer, H-bonds are effective in binary mixtures of methionine and alcohol solutions.

Partial molar volumes of components for all compositions can be calculated by using the Redlich-Kister coefficients in Eq. (3) at $298.15 \mathrm{~K}$, and the results are shown in Table 3. The parameters $A_{i}$ in Eq. (3) are reported in Table 4 along with standard deviations, $\sigma$, calculated by using Eq. (4). The partial molar volumes at infinite dilution show a good agreement between the calculation method, Eq. (11) and Eq. (12), and the linear extrapolation method of Eq. (8) and (9). As can be seen in Table 3, partial molar volumes in ethanol + water and methanol + water mixtures were calculated as close to each other. From these values, it is understood that there are stronger hydrogen bonds and dipole interactions between the sulfur in the structure of the amino acid and water molecules.

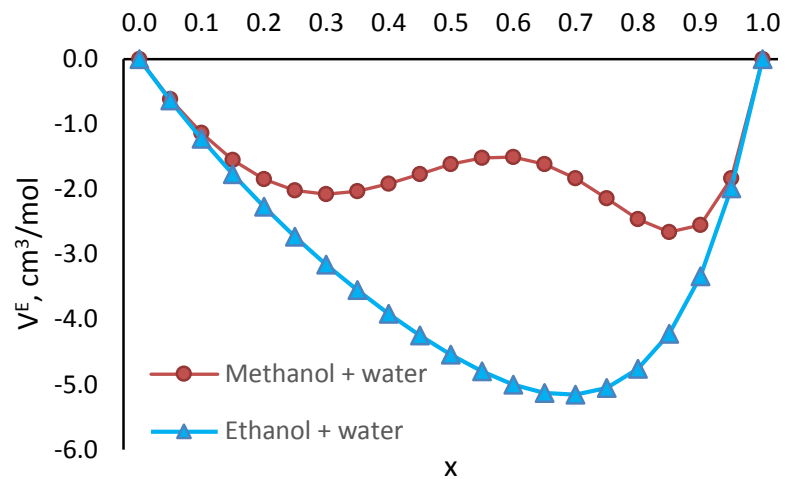

Figure 3. L-Excess molar volumes of methionine + (ethanol+water) and methionine $+($ methanol + water $)$ solutions at $298.15 \mathrm{~K}$.

From Eq. (13) and Eq. (14), excess apparent molar volumes values of methanol + water (1) and ethanol + water (2) solutions of methionine in infinitely dilute solutions were found $V_{1}^{\infty}=149.73 \mathrm{~cm}^{3} / \mathrm{mol}$ and $V_{2}^{\infty}=149.50 \mathrm{~cm}^{3} / \mathrm{mol}$ at $298.15 \mathrm{~K}$, respectively. Similarly, it was found to be $V_{1}^{\infty}=144.74 \mathrm{~cm}^{3} / \mathrm{mol}$ and $V_{2}^{\infty}=149.50 \mathrm{~cm}^{3} / \mathrm{mol}$ from the linear extrapolation of Eq. (20) and Eq. (21). However, it can be said that the value found by interpolation is more reliable.

The change in volumetric properties of a binary mixture of methionine + alcohol can be explained by chemical interactions that occur in the form of charge-transfer interactions, hydrogen bonds and dipole-dipole interactions. Charge transfer bonds are formed between the electron donor amine group and the electron acceptor sulfur and carboxyl groups in the methionine structure. Strong hydrogen bonds and dipole-dipole interactions occur between the N, S atoms in the side chain molecules of methionine and the -OH group of the water molecule. Dipole-dipole interactions occur due to the dipole moment difference. The dipole moments of water, ethanol and methanol molecules are $1.85 \mathrm{D}, 1.67 \mathrm{D}$ and $1.69 \mathrm{D}$, respectively [21,22].

Table 3. Apparent Molar Volumes and Partial Molar Volumes of Methionine + (Ethanol + Water) and (Methanol + Water) Solutions at $298.15 \mathrm{~K}$.

\begin{tabular}{|c|c|c|c|c|c|c|c|c|}
\hline \multirow[b]{2}{*}{$x$} & \multicolumn{4}{|c|}{$\begin{array}{l}\text { Apparent molar volumes, } \\
\mathrm{V}_{\phi},\left(\mathrm{cm}^{3} / \mathrm{mol}\right)\end{array}$} & \multicolumn{2}{|c|}{$\begin{array}{l}\text { Partial Molar Volume, } \\
\mathrm{V}_{\mathrm{i}}\left(\mathrm{cm}^{3} / \mathrm{mol}\right)\end{array}$} & \multicolumn{2}{|c|}{$\begin{array}{l}\text { Excess partial Molar Volume, } \mathrm{V}_{\mathrm{i}}^{\mathrm{E}} \\
\left(\mathrm{cm}^{3} / \mathrm{mol}\right)\end{array}$} \\
\hline & $\begin{array}{l}\text { Methanol } \\
+ \text { Water }^{\mathrm{a}}\end{array}$ & $\begin{array}{l}\text { Ethanol } \\
+ \text { Water }^{\mathrm{b}}\end{array}$ & $\begin{array}{l}\text { Methanol } \\
+ \text { Water }\end{array}$ & $\begin{array}{l}\text { Ethanol } \\
+ \text { Water }^{\mathrm{d}}\end{array}$ & $\begin{array}{l}\text { Methanol } \\
+ \text { Water }\end{array}$ & $\begin{array}{l}\text { Ethanol } \\
+ \text { Water }\end{array}$ & $\begin{array}{l}\text { Methanol } \\
+ \text { Water }\end{array}$ & $\begin{array}{l}\text { Ethanol } \\
+ \text { Water }\end{array}$ \\
\hline 0.00 & 147.97 & 147.43 & 149.75 & 149.46 & 149.73 & 149.46 & -0.01 & 0.00 \\
\hline 0.05 & 146.18 & 147.93 & 115.58 & 147.87 & 149.74 & 149.47 & 1.70 & -0.47 \\
\hline 0.10 & 145.23 & 148.72 & 123.59 & 146.40 & 149.74 & 149.47 & 2.61 & -1.23 \\
\hline 0.15 & 145.20 & 149.74 & 132.12 & 145.01 & 149.75 & 149.47 & 2.64 & -2.20 \\
\hline 0.20 & 146.09 & 150.91 & 140.77 & 143.64 & 149.75 & 149.47 & 1.80 & -3.33 \\
\hline 0.25 & 147.82 & 152.20 & 149.20 & 142.23 & 149.75 & 149.46 & 0.14 & -4.56 \\
\hline 0.30 & 150.29 & 153.55 & 157.16 & 140.71 & 149.75 & 149.46 & -2.22 & -5.86 \\
\hline 0.35 & 153.31 & 154.95 & 164.37 & 139.02 & 149.75 & 149.46 & -5.12 & -7.20 \\
\hline 0.40 & 156.68 & 156.38 & 170.60 & 137.10 & 149.75 & 149.46 & -8.34 & -8.57 \\
\hline 0.45 & 160.14 & 157.84 & 175.63 & 134.88 & 149.75 & 149.46 & -11.65 & -9.96 \\
\hline 0.50 & 163.40 & 159.33 & 179.28 & 132.29 & 149.75 & 149.46 & -14.77 & -11.39 \\
\hline 0.55 & 166.18 & 160.90 & 181.44 & 129.27 & 149.75 & 149.46 & -17.43 & -12.89 \\
\hline 0.60 & 168.28 & 162.58 & 182.15 & 125.78 & 149.75 & 149.46 & -19.44 & -14.50 \\
\hline 0.65 & 169.57 & 164.45 & 181.56 & 121.80 & 149.75 & 149.46 & -20.68 & -16.29 \\
\hline 0.70 & 170.12 & 166.59 & 180.04 & 117.35 & 149.75 & 149.46 & -21.20 & -18.34 \\
\hline 0.75 & 170.16 & 169.12 & 178.07 & 112.62 & 149.75 & 149.46 & -21.24 & -20.76 \\
\hline 0.80 & 170.15 & 172.19 & 176.29 & 108.09 & 149.75 & 149.46 & -21.23 & -23.69 \\
\hline 0.85 & 170.78 & 175.98 & 175.44 & 105.20 & 149.75 & 149.46 & -21.84 & -27.33 \\
\hline 0.90 & 173.06 & 180.76 & 176.43 & 108.82 & 149.75 & 149.47 & -24.01 & -31.90 \\
\hline 0.95 & 178.45 & 186.87 & 180.45 & 145.04 & 149.75 & 149.46 & -29.17 & -37.75 \\
\hline 1.00 & 189.42 & 194.77 & 189.42 & 194.77 & 149.75 & 149.53 & -39.68 & -45.24 \\
\hline
\end{tabular}

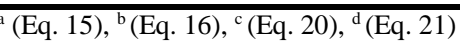


Tablo 4. The Values of Redlich-Kister Coefficients for The Studied Binary Mixtures of at $298.15 \mathrm{~K}$ and at Under The Atmospheric Pressure

\begin{tabular}{lll}
\hline Mixture & Metanol + Water & Ethanol + Water \\
\hline $\mathrm{A}_{0}$ & 0,5908 & 0,1995 \\
$\mathrm{~A}_{1}$ & $-0,5940$ & $-0,0924$ \\
$\mathrm{~A}_{2}$ & $-0,0172$ & $-0,2116$ \\
$\mathrm{~A}_{3}$ & 0,0064 & 0,0415 \\
$\mathrm{~A}_{4}$ & $-0,0005$ & $-0,0027$ \\
$\mathrm{~A}_{5}$ & 0,0000 & 0,0001 \\
$\sigma$ & 7,7400 & 15,07 \\
\hline
\end{tabular}

\section{Conclusion}

In this study, the intermolecular interactions of methionine in aqueous alcohol solutions at $298.15 \mathrm{~K}$ were tried to be understood from the density, apparent molar volume, partial molar volume and excess molar properties. Since methionine, which has a strong polar structure, has strong functional groups such as sulfur, carboxyl, amine, and strong interactions between water molecules, the effect of increasing alcohol concentration on these interactions was weak. As the ethanol concentration increases, the volumetric changes are slightly more pronounced compared to methanol. Hydrogen bonds, dipole interactions and charge transfer complexes are stronger than structural interactions in the molecular behaviour of amino acids in solutions.

The volumetric properties of amino acids in water and alcohol are extremely important for understanding their interactions with the structures surrounding the proteins. Understanding the solvent-soluble, solute-soluble interactions of amino acids, which are the building blocks of proteins, is important in terms of understanding the behaviour of amino acids in biochemical reactions. As it can be understood from the volumetric properties of methionine, depending on the chemical structure of biological systems, interactions deviating from the ideal state occur with the amino acid due to physical and chemical effects such as hydrogen bonds, dipole interactions, charge-transfer interactions. These interactions, which occur due to these chemical and physical effects, can trigger biological events depending on the environment. For this reason, volumetric properties will give an idea for a better understanding of the behavior of compounds such as methionine, which have an important role in biological events, in solutions.

$\begin{array}{ll}\text { Nomenclature } \\ A_{i} \quad \text { Redlich-Kister equation Coefficients } \\ M_{i} \quad \text { Molar masses of components, } \\ \rho_{i} \quad \text { Densities of pure components } \\ \rho & \text { Density of the mixture, } \\ \sigma\left(V^{E}\right) & \text { Standart deviation } \\ V & \text { Molar volume of the solution } \\ V_{\phi} & \text { Apparent molar volume } \\ V_{\emptyset, i}^{E} & \text { Excess apparent molar volumes at infinite dilution, } \\ V_{i}^{0} & \text { Molar volumes of pure components } \\ V_{i}^{E} & \text { Excess partial molar volume, } \\ V_{i}^{\infty} & \text { Partial molar volumes at infinite dilution } \\ V_{i}^{0} & \text { Molar volume of the pure component } \\ V_{\text {exp }}^{E} & \text { Experimental excess molar volume } \\ V_{\text {cal }}^{E} & \text { Calculated excess molar volume } \\ x_{i} & \text { Mole fractions of components }\end{array}$

$x \quad$ Mole fractions of the mixture

\section{References}

[1] J. T. Brosnan, M. E. Brosnan, R. F.P. Bertolo and J. A. Brunton, "Methionine: A metabolically unique amino acid," Livestock Science, 112(1-2): 2-7, 2007.

[2] G. Wu, Y. Z. Fang, S. Yang, J. R. Lupton and N. D. Turner, "Glutathione metabolism and its implications for health," J Nutr. 134(3): 489-92, 2004.

[3] M. H. Stipanuk and I. Ueki, “ Dealing with methionine/homocysteine sulfur: cysteine metabolism to taurine and inorganic sulfur," Journal of inherited metabolic disease, 34(1): 17-32, 2011.

[4] J. C. Heiby, B. Goretzki, and C. M. Johnson et al. "Methionine in a protein hydrophobic core drives tight interactions required for assembly of spider silk," Nat Commun 10, 4378, 2019.

[5] S. Ravanel, B. Gakière, D. Job and R. Douce, "The specific features of methionine biosynthesis and metabolism in plants," Proc. Natl. Acad. Sci. 95(13): 7805-7812, 1998.

[6] S. M. Phillips, "A brief review of critical processes in exercise-induced muscular hypertrophy," Sports Med. 44(1): 71-77, 2014.

[7] J. T. Brosnan and M. E. Brosnan, "The SulfurContaining Amino Acids: An Overview," The Journal of Nutrition, 136(6): 1636S-640S, 2006.

[8] T. Zhang, Z. Li, Y. Wang, C. Li, B. Yu, X. Zheng, L. Jiang, J. Gong, "Determination and correlation of solubility and thermodynamic properties of 1methionine in binary solvents of water+(methanol, ethanol, acetone)," The Journal of Chemical Thermodynamics, 96: 82-92, 2016.

[9] E. Yu. Tyunina, V. I. Smirnov and G. N. Tarasova, "Thermodynamic Properties of L-Methionine and Nicotinic Acid in an Aqueous Buffer Solution", Russian Journal of Physical Chemistry A, 94(11): 2238-2243, 2020.

[10] F. I. El-Dossoki, "Phase Diagrams, Molal Volumes and Polarizabilities of (Lysine, Methionine Amino Acids-Alcohol-Water) Tri-Component Systems," International Research Journal of Pure \& Applied Chemistry, 16(2): 1-11, 2018

[11] F. I. El-Dossoki, "Phase Diagrams, Molal Volumes and Polarizabilities of (Lysine, Methionine Amino Acids-Alcohol-Water)Tri-Component Systems,"International Research Journal of Pure \& Applied Chemistry, 16(2): 1-11, 2018.

[12] M. Mokhtarpour and H. Shekaari, "Measurement and correlation of thermophysical properties in aqueous solutions of some novel bio-based deep eutectic solvents (lactic acid/amino acids) at $\mathrm{T}=(298.15$ to 313.15) K," J. Chem. Thermodynamics, 144, 106051, 2020.

[13] A. Shalmashi and F. Amani, "Densities And Excess Molar Volumes For Binary Solution Of Water + Ethanol, + Methanol And + Propanol From (283.15 To 313.15) K," Latin American Applied Research, 44: 163-166, 2014. 
[14] A. Ali, A. K. Nain, V. K. Sharma and S. Ahmad, "Molecular interactions in binary mixtures of tetrahydrofuran with alkanols (C6, C8, C10): an ultrasonic and volumetric study," Indian J Pure Appl Phys, 42: 666-673, 2004.

[15] H. Iloukhani, M. Rezaei-Sameti and J. Basiri-Parsa, "Excess molar volumes and dynamic viscosities for binary mixtures of toluene ? n-alkanes (C5-C10) at T = 298.15 K, comparison with Prigogine-Flory-Patterson theory. J Chem Thermodyn. 38: 975-982, 2006.

[16] U. S. Vural, "Excess molar volumes and viscosities of binary mixtures of epichlorohydrine and alcohols," Russian Journal of Physical Chemistry A, 79: 10961101, 2005.

[17] U. S. Vural, V. Muradoglu and S. Vural, "Excess molar volumes, and refractive index of binary mixtures of glycerol + methanol and glycerol + water at $298.15 \mathrm{~K}$ and $303.15 \mathrm{~K}, "$ Bulletin of the Chemical Society of Ethiopia, 25: 111, 2011

[18] W. A. A. Ddamba, "Excess and Partial Molar Volumes for [Difurylmethane+Acetonitrile] Binary Mixtures in the Temperature Range: 288.15-323.15 K," Asian Journal of Chemistry, 21(4): 3057-3067, 2009.

[19] F. Allal, A. Dahmani, B. Saidat, "Volumetric Properties of Binary Mixtures of 1-Ethyl-3methylimidazolium ethyl phosphonate + Methanol or 2-Propanol," International Journal of Thermodynamics (IJoT), 22(3): 128-136, 2019.

[20] H. Iloukhani and H. Bahrami, "Excess Molar Volumes and Partial Molar Volumes For Binary Mixtures of Water with 1,2-Ethanediol, 1,2-Propanediol, and 1,2Butanediol at 293.15, 303.15 and $313.15 \mathrm{~K}$," Phys. Chem. Liq., 38: 103-111, 2004.

[21] T. Zhu and T. V. Voorhis, "Understanding the Dipole Moment of Liquid Water from a Self-Attractive Hartree Decomposition," The Journal of Physical Chemistry Letters, 12 (1): 6-12, 2021.

[22] F. C Frank, "Dipole Induction and the Solvent Effect in Dipole Moment Measurements," Proceedings of the Royal Society of London. Series A, Mathematical and Physical Sciences, 152 (875): 171-196, 1935. 